different seasonal behaviour of $N$ in the two hemispheres is believed to be due to a difference in the seasonal variation of the phase of the tides in the two hemispheres.

\section{Council for Scientific and Industrial Research}

D. F. Martirn. and Commonwealth Observatory, Canberra.

\section{Magnetic Properties of some Paramagnetic Crystals at Low Temperatures}

Measurements have been made on the principal magnetic susceptibilities of single crystals of a large number of paramagnetic salts of the iron group, from room temperature down to about $80^{\circ} \mathrm{K}$. A detailed discussion of the results on the basis of the crystalline field theory will shortly be published elsewhere. According to this theory, the deviations from the simple behaviour to be expected of the free ions are attributed to the Stark splitting of the energy-levels of the paramagnetic ion under the influence of the strong and generally asymmetric electric field that occurs in the neighbourhood of the paramagnetic ions in these crystals, due to the negatively charged atoms that surround these ions. The following are some of the main results obtained.

In manganous and ferric salts in which the paramagnetic ion is in the $S$-state, the anisotropies are negligibly small, and the temperature variation of the susceptibility almost exactly follows the Curie law $\chi=C / T$.

In chromic salts also this is so, even though the $\mathrm{Cr}^{+++}$ion is not in the $S$-state. This is due to the fact that the lowest level in the Stark pattern of $\mathrm{Cr}^{+++}$is a singlet, and it is widely separated from the upper levels; the singlet level, however, has a $(2 s+1)$-fold spin-degeneracy.

The nickel salts are very similar to the chromic salts in their magnetic behaviour, except that the spin-orbit coupling in $\mathrm{Ni}^{++}$is much greater than in $\mathrm{Cr}^{+++}$, and this conduces to a much larger deviation from the $S$-state behaviour than in $\mathrm{Cr}^{+++}$; in other words, it leads to an appreciable magnetic anisotropy and deviation from the Curie law. These deviations are utilized to calculate the crystal field constants, and it is found that not only the cubic part of the field which is predominant, but even the rhombic part which is small, is nearly the same in most of the nickel salts, and they are practically independent of temperature.

In both $\mathrm{Co}^{++}$and $\mathrm{Fe}^{++}$salts, owing to the groundlevel being a triplet, the magnetic properties are more sensitive to the rhombic part of the field than in the nickel salts. An estimate has been made of the rhombic part of the field.

The copper salts, as judged by their magnetic properties, fall into three distinct classes. In the first class all the principal susceptibilities follow the Curie law, but with different Curie constants; that is, the effective moments are different for different directions, but all of them are practically independent of temperature. To this class belong the Tutton salts. In the second class the principal moments are nearly the same as before, but they all decrease with the lowering of temperature, slowly at first and rapidly at later stages. The double chloride of copper and ammonium is a typical example of this class. To the third class belongs cupric acetate monohydrate, in which the principal moments are all very low even at room temperature and decrease rapidly as the temperature is lowered. The three classes correspond to the non-cubic part of the crystalline field, being very different, lowest in the first class and highest in the third.

The influence of covalent binding on the strength of the electric field, and ultimately on the breaking of the Russell-Saunders coupling not only between the spin and orbital moments, but also between the spins of the different electrons of the ion, and its effect on the magnetic properties of the ion, have been discussed.

Though in the majority of the crystals the magnetio axes do not change their directions in the range of temperatures studied, the change is appreciable in some crystals, the largest change observed being $7^{\circ}$.

There is an axis of magnetic symmetry in nickel and ferrous salts, even though the crystal structure does not lead to it.

My thanks are due to Prof. K. S. Krishnan for his interest in this work, and to the Indian Association for the Cultivation of Science, Calcutta, for facilities for carrying out the experiments.

\section{Larmini Street, P.O. Wari, Dacca. Jan. 15. \\ Bhagawati Charan GuHa. \\ Theory of Viscosity of Concentrated Suspensions}

For infinitely diluted suspensions of rigid spheres, Einstein ${ }^{1}$ derived on a rigorously theoretical basis the formula

$$
\eta=\eta_{0}(1+k c),
$$

where $\eta$ is the viscosity of the suspension, $\eta_{0}$ is the viscosity of the pure solvent, $c$ is the concentration by volume and $k=2 \cdot 5$ is a constant. It has been shown that (1) is also valid for non-spherical particles, the factor $k$ being a function of their shape, rigidity and Brownian movement. For elongated rigid particles, $k>2 \cdot 5$.

If it is assumed that the suspension behaves hydro. dynamically with respect to an additional particle as a homogeneous medium, it is possible to derive a theoretical viscosity formula for higher concentrations. Let us add a particle of a volume $d V$ to a suspension of viscosity $\eta$, containing already a volume $V$ of similar particles in a volume $A$ of the pure liquid. Then the increase of the viscosity should be

$$
\eta+d \eta=\eta\left(1+k \frac{d V}{A+V}\right), \quad . .
$$

or after a slight rearrangement

$$
\frac{d \eta}{\eta}=k \frac{d V}{A+V}, \quad \cdot . \cdot
$$

which can be easily integrated, giving

$$
\eta=\eta_{0}(1+\nabla / A)^{k}=\eta_{0}(1-c)^{-k} .
$$

If $c=0, \eta=\eta_{0}$, so that the integration constant $\eta_{0}$ is the viscosity of the pure liquid.

The assumption of hydrodynamical homogeneity of the suspension holds only if the particles are sufficiently far apart, so that their mutual interactions other than a general increase of viscosity are neglig. 